\title{
SOME PROPERTIES OF $p$-VALENT ANALYTIC FUNCTIONS INVOLVING CHO-KWON-SRIVASTAVA INTEGRAL OPERATOR
}

\author{
K. I. NOOR, S. Z. H. BUKHARI, M. ARIF AND M. NAZIR
}

Abstract. In this paper, we introduce a subclass of $p$-valent functions involving Cho-KwonSrivastava integral operator. We study some interesting results including inclusion relations, convoltion with convex functions and integral preserving property for this class.

Mathematics subject classification (2010): 30C45, 30C50.

Keywords and phrases: Convolution, subordination, Cho-Kwon-Srivastava integral operator.

\section{REFERENCES}

[1] B. C. CARLson, B. D. ShafFer, Starlike and prestarlike hypergeometric functions, SIAM J. Math. Anal., 15 (4) (1984), 737-745.

[2] N. E. ChO, O. S. KWON, H. M. SRIVASTAVA, Inclusion relationships and argument properties for certain subclasses of multivalent functions associated with a family of linear operators, J. Math. Anal. Appl., 292 (2004), 470-483.

[3] J. H. ChOI, M. SAigo, H. M. SRIVASTAVA, Some inclusion properties of a certain family of integral operators, J. Math. Anal. Appl. 276 (2002), 432-445.

[4] P. Eenigenburg, S. S. Miller, P. T. Mocanu, M. O. Reade, On a Briot-Bouquet differential subordination, Gen. Math. 3, Int. Series of Num. Math., Birkhäuser Verlag, Basel, 64 (1983), 339-348; see also Rev. Roumaine Math. Pures Appl., 29 (1984), 567-573.

[5] H. IRMAK, R. K. RAINA, The starlikeness and convexity of multivalent functions involving certain inequalities, Rev. Mat. Complut., 16 (2) (2003), 391-39.

[6] Y. C. KIM, J. H. CHOI, T. SUGAWA, Coefficient bounds and convolution properties for certain classes of close-to-convex functions, Proc. Japan Acad. Ser. A Math. Sci. 76 (2000), 95-98.

[7] J.-L. LiU, The Noor integral and strongly starlike functions, J. Math. Anal. Appl,. 261 (2001), 441447.

[8] W. MA, D. Minda, An internal geometric characterization of strongly starlike functions, Ann. Univ. Mariae Curie-Sklodowska Sect. A, 45 (1991), 89-97.

[9] S. S. Miller, P. T. Mocanu, Differential Subordinations: Theory and Applications, Pure Appl. Math., Vol. 225, Dekker, New York, 2000.

[10] K. I. Noor, On new classes of integral operators, J. Natur. Geom., 16 (1999), 71-80.

[11] K. I. Noor, On classes of analytic functions defined by convolution with incomplete beta functions, J. Math. Anal. Appl., 307 (2005), 339-349.

[12] K. I. Noor, On some applications of certain integral operators, Appl. Math.Comput., 188 (2007), 814-823.

[13] K. I. Noor, S. Mustafa, B. Malik, On some classes of p-valent functions involving certain Carlson Shaffer operator, Appl. Math. Comput, 214 (2009), 336-341.

[14] K. I. Noor, M. A. Noor, On integral operators, J. Math. Anal. Appl., 238 (1999), 341-352.

[15] S. OwA, On the distortion theorems I, Kyungpook Math. J., 18 (1) (1978), 53-59.

[16] S. OWA, H. M. SRIVASTAVA, Univalent and starlike generalized hypergeometric functions, Canad. J. Math., 39 (5) (1987), 1057-1077.

[17] M. S. RoberTson, Multivalently starlike functions, Duke Math. J., 20 (1953), 539-549.

[18] St. Ruscheweyh, Convolutions in Geometric Function Theory, Les Presses de l'Univ. de Montreal, 1982.

[19] H. SAitoh, A linear operator and its applications of first order differential subordinations, Math. Japon., 44 (1996), 31-38. 
[20] J. SoKó£, L. T. Spelina, Convolution properties for certain classes of multivalent functions, J. Math. Anal. Appl. 337 (2008), 1190-1197.

[21] H. M. SRIVASTAVA, S. OWA, An application of the fractional derivative, Math. Japon., 29 (3) (1984), 383-389.

[22] T. YAGUCHI, The radii of starlikeness and convexity for certain multivalent functions, in: $\mathrm{H}$. M. Srivastava, S. Owa (Eds.), Current Topics in Analytic Function Theory, World Scientific, Singapore, 1992, 375-386. 